\title{
On a generic class of regular one-parametric variational inequalities.
}

\author{
Gómez Bofill, Walter \\ Institut für Mathematik, Humboldt Universität zu Berlin, \\ Unter den Linden 6, D-10099, Berlin, Germany
}

\begin{abstract}
In this paper the regularity of one-parametric optimization problems in the sense of Jongen, Jonker and Twilt is extended to oneparametric variational inequalities. The five singularities are defined in this context and suitable indices are described around them. Many of the local properties of the singularities are also proved for this case.

The generic property of the defined class of regular one-parametric variational inequalities is proved and a corresponding linear quadratic perturbation result is stated.
\end{abstract}

Keywords: one-parametric variational inequalities, singularities, genericity

\section{Introduction.}

Let $F: M \rightarrow \mathbb{R}^{n}$ be a mapping and $M \subset \mathbb{R}^{n}$ be a closed set defined as

$$
M=\left\{x \in \mathbb{R}^{n} \mid H(x)=0, G(x) \geq 0\right\},
$$

where $(H, G): \mathbb{R}^{n} \rightarrow \mathbb{R}^{m+p}$. The variational inequality problem is defined as follows: Find some point $x \in M$ such that

$$
F(x)(y-x) \geq 0, \quad \forall y \in M
$$


This problem has found wide application as an adequate mathematical framework for a number of economic, game-theoretic and equilibrium problems. For extensive surveys we cite e.g. [5] and [13].

Special cases of variational inequalities are for example equality systems $\left(M=\mathbb{R}^{n}\right)$, nonlinear complementarity problems $\left(M=\mathbb{R}_{+}^{n}\right)$, and convex minimization problems $(F=\nabla f)$.

If $M$ is convex ( $-g_{j}$ are convex and $h_{i}$ are affine linear) the solutions of the variational inequalities can be characterized, under constraint qualification, by a suitable version of the well-know KKT-system. This convex case have been intensively studied and various reformulations of the variational inequality problem are given, for example as a system of equations (e.g. the normal map), as a generalized equation (via the normal cone) and as an optimization problem (constrained and unconstrained). Many of the solution approaches proposed are very similar and base on the use of generalized Newton methods for nonsmooth equations (see e.g. [13], [5], [6], [3], [2], [8]).

A further approach was given recently (see e.g. [15], [18], [19] [17]), which base on the use of continuation methods. In this paper we are also interested in the application of such continuation techniques for the solution of variational inequalities. Our main purpose is to extend the well-known regularity of one-parametric optimization problems in the sense of Jongen, Jonker and Twilt (see [11]) to the case of one-parametric variational inequalities. This regularity deals with the local structure of the solution sets of one-parametric optimization problems and is an important theoretical issue, for instance by the so called path-following methods with jumps (see e.g. [4]).

The paper is organized as follows. In the second section we redefine the singularities, which characterize the regularity in the sense of Jongen, Jonker and Twilt, in the case of one-parametric varational inequalities and state the most important local properties. In the third section other properties are stated, for instance the generic character of the regular problems.

\section{One-parametric variational inequalities.}

Let us consider variational inequalities $(F(x, t), H(x, t), G(x, t))$ depending of a one-dimensional real parameter $t$. Such problems are defined by its data $(F, H, G): \mathbb{R}^{n+1} \rightarrow \mathbb{R}^{n+m+p}$ and will also be denoted by $V I(F, H, G)$. The corresponding feasible set $M \subset \mathbb{R}^{n+1}$ will also be denoted by $M(H, G)$.

Let us denote $z=(x, t) \in \mathbb{R}^{n+1}, I=\{1, \ldots, m\}$ and $J=\{1, \ldots, p\}$. For 
a given parameter value $t$ the notation $V I(F, H, G)(t)$ and $M(H, G)(t) \subset \mathbb{R}^{n}$ (or simply $M(t)$ ) state for the corresponding variational inequality and its feasible set.

Many well-known concepts from optimization theory concerning feasible sets apply also to variational inequalities, for instance constraint qualifications as LICQ or MFCQ, active index set, etc.

A feasible point $z \in M(H, G)$ of the $V I(F, H, G)$ is a critical point if there exist $(\lambda, \mu) \in \mathbb{R}^{m+\left|J_{0}(z)\right|}$ satisfying the following relation

$$
\mathrm{L}(z, \lambda, \mu)=0,
$$

where $\mathrm{L}(z, \lambda, \mu)=F(z)-\sum_{i=1}^{m} \lambda_{i} D_{x} h_{i}(z)-\sum_{j \in J_{0}(z)} \mu_{j} D_{x} g_{j}(z)$. If $\mu_{j} \geq 0$, $\forall j \in J_{0}(z)$ then $z$ is called a stationary point.

If the vectors $\left\{F(z), D_{x} h_{i}(z), D_{x} g_{j}(z), i \in I, j \in J_{0}(z)\right\}$ are linearly dependent, $z$ is defined as a generalized critical point, shortly g.c. point (see [11]).

We consider in this paper mainly the sets

$$
\begin{aligned}
\Sigma_{g c} & =\left\{z \in \mathbb{R}^{n+1} \mid z \text { is a g.c. point of } V I(F, H, G)\right\} \\
\Sigma_{\text {stat }} & =\left\{z \in \mathbb{R}^{n+1} \mid z \text { is a stationary point of } V I(F, H, G)\right\}
\end{aligned}
$$

If necessary, the notation $\Sigma(F, H, G)$ will be used for recalling the data.

\section{Definition 1}

A g.c. point $\bar{z}$ of $V I(F, H, G)$ is called non-degenerated if the LICQ holds and the following conditions are fulfilled:

VU-ND1 : The uniquely determined solution $(\bar{\lambda}, \bar{\mu}) \in \mathbb{R}^{m+\left|J_{0}(\bar{z})\right|}$ of (D) satisfies $\bar{\mu}_{j} \neq 0, \forall j \in J_{0}(\bar{z})$.

VU-ND2 : The matrix $\left.D_{x} \mathrm{~L}(\bar{z}, \bar{\lambda}, \bar{\mu})\right|_{T_{\bar{x}} M(\bar{t})}$ is nonsingular.

Here $T_{\bar{x}} M(\bar{t})$ is the tangential space to the set $M(\bar{t})$ at the point $\bar{x}$ and $\left.D_{x} \mathrm{~L}(\bar{z}, \bar{\lambda}, \bar{\mu})\right|_{T_{\bar{x}} M(\bar{t})}$ is a matrix of the form $V^{T} D_{x} \mathrm{~L}(\bar{z}, \bar{\lambda}, \bar{\mu}) V$, where the columns from $V$ build a linear basis for $T_{\bar{x}} M(\bar{t})$. In general, $D_{x} \mathrm{~L}(\bar{z}, \bar{\lambda}, \bar{\mu})$ is not symmetric. The $\operatorname{sign}\left[\operatorname{det}\left(V^{T} D_{x} \mathrm{~L}(\bar{z}, \bar{\lambda}, \bar{\mu}) V\right)\right]$ is independent of the possible selection of $V$. Let us define for $K \subset J$ the following mapping

$$
\mathrm{H}_{K}(z, \lambda, \mu)=\left(\begin{array}{c}
F(z)-\sum_{i=1}^{m} \lambda_{i} D_{x} h_{i}(z)-\sum_{j \in K} \mu_{j} D_{x} g_{j}(z) \\
H(z) \\
G_{K}(z)
\end{array}\right) .
$$




\section{Remark 1}

Under the LICQ the Condition VU-ND2 is equivalent to the regularity from $D_{(x, \lambda, \mu)} \mathrm{H}_{J_{0}}(z, \lambda, \mu)$. The set of critical points is given locally under VU-ND1 by the projection onto the z-components of the zeros of $\mathrm{H}_{J_{0}}$ and, therefore, due to the non-degeneracy it builts a smooth one-dimensional manifold that is parametrizable in $t$.

At a non-degenerated g.c. point the $L I$ and $L C I$ are uniquely determined (number of negative and positive entries of $\bar{\mu}) . \operatorname{sign}\left[\operatorname{det}\left(\left.D_{x} \mathrm{~L}(\bar{z}, \bar{\lambda}, \bar{\mu})\right|_{T_{\bar{x}} M(\bar{t})}\right)\right]$ is also uniquely determined. Around the different singularities we are then going to describe the changes of the triple

$$
\left(L I, L C I, \operatorname{sign}\left[\operatorname{det}\left(\left.D_{x} \mathrm{~L}(z, \lambda, \mu)\right|_{T_{x} M(t)}\right)\right]\right)
$$

Taking into account the relation

$$
\operatorname{sign}\left[\operatorname{det}\left(\left.D_{x} \mathrm{~L}(\bar{z}, \bar{\lambda}, \bar{\mu})\right|_{T_{\bar{x}} M(\bar{t})}\right)\right]=\operatorname{sign}\left[\operatorname{det}\left(D_{(x, \lambda, \mu)} \mathrm{H}_{J_{0}}(\bar{z}, \bar{\lambda}, \bar{\mu})\right)\right],
$$

we define $\operatorname{sign}\left(\operatorname{det}\left(\left.D_{x} \mathrm{~L}(\bar{z}, \bar{\lambda}, \bar{\mu})\right|_{T_{\bar{x}} M(\bar{t})}\right)\right)=+1$ for the case of $T_{\bar{x}} M(\bar{t})=\{0\}$.

\subsection{Singularities and local properties.}

A g.c. point $\bar{z}$ is of Type 1 if it is non-degenerated as in Definition 1.

\section{Remark 2}

In the neighbourhood of a g.c. point $\bar{z}$ of Type 1 the points of $\Sigma_{g c}$ are nondegenerated and the triple (3) remain constant.

Definition 2 (see also [11])

A g.c. point $\bar{z}$ of $V I(F, H, G)$ is of Type 2 if the following conditions hold:

Type2: 1-VU : The LICQ is fulfilled

Type2: 2-VU : Let $(\bar{\lambda}, \bar{\mu}) \in \mathbb{R}^{m+\left|J_{0}(z)\right|}$ solve (D), then there exists exactly one index $l \in J_{0}(\bar{z})$, such that $\bar{\mu}_{l}=0$, and $\bar{\mu}_{k} \neq 0, \forall k \in J_{0}(\bar{z}) \backslash\{l\}$.

Type2: 3-VU : $\left.D_{x} \mathrm{~L}(\bar{z}, \bar{\lambda}, \bar{\mu})\right|_{T_{\bar{x}} M(\bar{t})}$ is nonsingular.

Type2: 4-VU : $\left.D_{x} \mathrm{~L}(\bar{z}, \bar{\lambda}, \bar{\mu})\right|_{T_{\bar{x}}^{+} M(\bar{t})}$ is nonsingular, where

$$
\begin{aligned}
& T_{\bar{x}}^{+} M(\bar{t})=\left\{\xi \in \mathbb{R}^{n} \mid D_{x} H(\bar{z}) \cdot \xi=0, D_{x} G_{J_{0}^{+}(\bar{z})}(\bar{z}) \cdot \xi=0\right\} \\
& \text { and } J_{0}^{+}(\bar{z})=\left\{j \in J_{0}(\bar{z}) \mid \bar{\mu}_{l} \neq 0\right\}=J_{0}(\bar{z}) \backslash\{l\} .
\end{aligned}
$$


Type2: 5-VU : Let us denote

- $\Pi_{+}=\left(H, G_{J_{0}^{+}(\bar{z})}\right)^{T}$, and

- $W$ is a matrix columns of which form a basis of $T_{\bar{x}}^{+} M$.

It holds that $\gamma \neq 0$, where

$$
\begin{aligned}
\gamma & =D_{x} g_{l}(\bar{z})(\alpha+\beta)+D_{t} g_{l}(\bar{z}) \\
\alpha & =-\left(\left(D_{x} \Pi_{+}(\bar{z})\right)^{\dagger}\right)^{T} \cdot D_{t} \Pi_{+}(\bar{z}), \\
\beta & =-W\left(W^{T} \cdot D_{x} \mathrm{~L} \cdot W\right)^{-1} W^{T} \cdot\left[D_{x} \mathrm{~L} \cdot \alpha+D_{t} \mathbf{L}\right]
\end{aligned}
$$

and $B^{\dagger}=\left(B^{T} B\right)^{-1} B^{T}$ represent the Moore-Penrose Inverse of $B$.

Denote by $\left(x^{J_{0}^{+}}(t), t, \lambda^{J_{0}^{+}}(t), \mu^{J_{0}^{+}}(t)\right)\left(\operatorname{resp} .\left(x^{J_{0}}(t), t, \lambda^{J_{0}}(t), \mu^{J_{0}}(t)\right)\right)$ a $t$ parametrization for the zeros of $\mathrm{H}_{J_{0}^{+}}\left(\right.$resp. $\left.\mathrm{H}_{J_{0}}\right)$.

\section{Remark 3}

It is easy to see that, around $\bar{z}$, the set $\Sigma_{g c}$ is given by the curve $\left(x^{J_{0}}(t), t\right)$ and the feasible part of $\left(x_{0}^{J_{0}^{+}}(t), t\right)$. Type2: 4-VU implies that the tangent lines of the two curves are not parallel.

For the description of the change from the triple (3) we use the characteristic numbers $\operatorname{sign}(\gamma)$ and $\operatorname{sign}(\delta)$, where

$$
\delta=\operatorname{det}\left(\left.D_{x} \mathrm{~L}(\bar{z}, \bar{\lambda}, \bar{\mu})\right|_{T_{\bar{x}} M(\bar{t})}\right) \cdot \operatorname{det}\left(\left.D_{x} \mathrm{~L}(\bar{z}, \bar{\lambda}, \bar{\mu})\right|_{T_{\bar{x}}^{+} M(\bar{t})}\right) .
$$

\section{Definition 3}

Let $A$ be a matrix divided as follows $A=\left(\begin{array}{cc}B & C \\ D & E\end{array}\right)$, where $B$ and $E$ are quadratic. Let $B$ be regular. The matrix $S(A \mid B)=E-D B^{-1} C$ is called the Schur-complement of $B$ in $A$.

Lemma 1 (see [1 4])

Let $A$ be as in Definition 3. Then it holds:

1. $\operatorname{det}(A)=\operatorname{det}(E) \cdot \operatorname{det}(S(A \mid B))$.

2. If $A$ is symmetric, it follows: $\operatorname{In}(A)=\operatorname{In}(B)+\operatorname{In}(S(A \mid B))$. 
3. If $A$ is regular, its inverse is given by:

$$
A^{-1}=\left(\begin{array}{ll}
B^{-1}+B^{-1} \cdot C \cdot S(A \mid B)^{-1} \cdot D \cdot B^{-1} & -B^{-1} \cdot C \cdot S(A \mid B)^{-1} \\
-S(A \mid B)^{-1} \cdot D \cdot B^{-1} & S(A \mid B)^{-1}
\end{array}\right) .
$$

Here $\operatorname{In}(P)=(p(P), n(P), z(P))$ represents the so-called innertia triple of a symmetric quadratic matrix $P$, where $p(P)$ (resp. $n(P)$ and $z(P)$ ) means the number of positive (resp. negative or zero) eigenvalues of $P$.

\section{Proposition 1}

Let $\bar{z}$ be a g.c. point of Type 2 with vanishing multiplier $\bar{\mu}_{l}$. It holds then that $\operatorname{sign}(\gamma) \cdot \operatorname{sign}(\delta)=-\operatorname{sign}\left(\dot{\mu}_{l}^{J_{0}}(\bar{t})\right)$.

\section{Proof:}

Let the columns of the matrix $V_{1}$ build a basis for $T_{\bar{x}} M(\bar{t})$ and the columns of $V_{0}=\left[V_{1}: v_{1}\right]$ one for $T_{\bar{x}}^{+} M(\bar{t})$. It holds:

$$
\left.D_{x} \mathrm{~L}\right|_{T_{x}^{+} M(t)}=\left(\begin{array}{cc}
V_{1}^{T} D_{x} \mathrm{~L} V_{1} & V_{1}^{T} D_{x} \mathrm{~L} v_{1} \\
v_{1}^{T} D_{x} \mathrm{~L} V_{1} & v_{1}^{T} D_{x} \mathrm{~L} v_{1}
\end{array}\right) .
$$

Type2: 3-VU implies the regularity of $V_{1}^{T} D_{x} \mathrm{~L} V_{1}$. Lemma 1 (1.-) gives that $\operatorname{det}\left(\left.D_{x} \mathrm{~L}\right|_{T_{\bar{x}}^{+} M(\bar{t})}\right)=\operatorname{det}\left(\left.D_{x} \mathrm{~L}\right|_{T_{\bar{x}} M(\bar{t})}\right) \operatorname{det}\left(S\left(\left.D_{x} \mathrm{~L}\right|_{T_{\bar{x}}^{+} M(\bar{t})}\left|D_{x} \mathrm{~L}\right|_{T_{\bar{x}} M(\bar{t})}\right)\right)$, where

$$
\begin{aligned}
S\left(\left.D_{x} \mathrm{~L}\right|_{T_{\bar{x}}^{+} M(\bar{t})}\left|D_{x} \mathrm{~L}\right|_{T_{\bar{x}} M(\bar{t})}\right)= & v_{1}^{T} D_{x} \mathrm{~L} v_{1}- \\
& v_{1}^{T} D_{x} \mathrm{~L} V_{1}\left(V_{1}^{T} D_{x} \mathrm{~L} V_{1}\right)^{-1} V_{1}^{T} D_{x} \mathrm{~L} v_{1} .
\end{aligned}
$$

Consequently, it holds:

$$
\operatorname{sign}(\delta)=\operatorname{sign}\left(S\left(\left.D_{x} \mathrm{~L}\right|_{T_{\bar{x}}^{+} M(\bar{t})}\left|D_{x} \mathrm{~L}\right|_{T_{\bar{x}} M(\bar{t})}\right)\right) .
$$

Since the derivatives of the components $\left(H, G_{J_{0}^{+}}\right)$in $\mathrm{H}_{J_{0}^{+}}$and $\mathrm{H}_{J_{0}}$ coincide, it holds that $\dot{x}_{0}^{J_{0}^{+}}(\bar{t})-\dot{x}^{J_{0}}(\bar{t}) \in T_{\bar{x}}^{+} M(\bar{t})$. Then there exists $w_{0}$ with $\dot{x}^{J_{0}^{+}}(\bar{t})-$ $\dot{x}^{J_{0}}(\bar{t})=V_{0} w_{0}$. Multiplying the Jacobi-matrix of $\mathbf{L}$ from the left-hand side by $V_{0}$ we obtain that $V_{0}^{T} D_{x} \mathrm{~L} V_{0} w_{0}+\dot{\mu}_{l}^{J_{0}}(\bar{t}) V_{0}^{T} D_{x} g_{l}(\bar{z})=0$. Consequently,

$$
\dot{x}^{J_{0}^{+}}(\bar{t})-\dot{x}^{J_{0}}(\bar{t})=-\dot{\mu}_{l}^{J_{0}}(\bar{t}) \cdot V_{0}\left(V_{0}^{T} D_{x} L V_{0}\right)^{-1} V_{0}^{T} D_{x}^{T} g_{l}(\bar{z}) .
$$




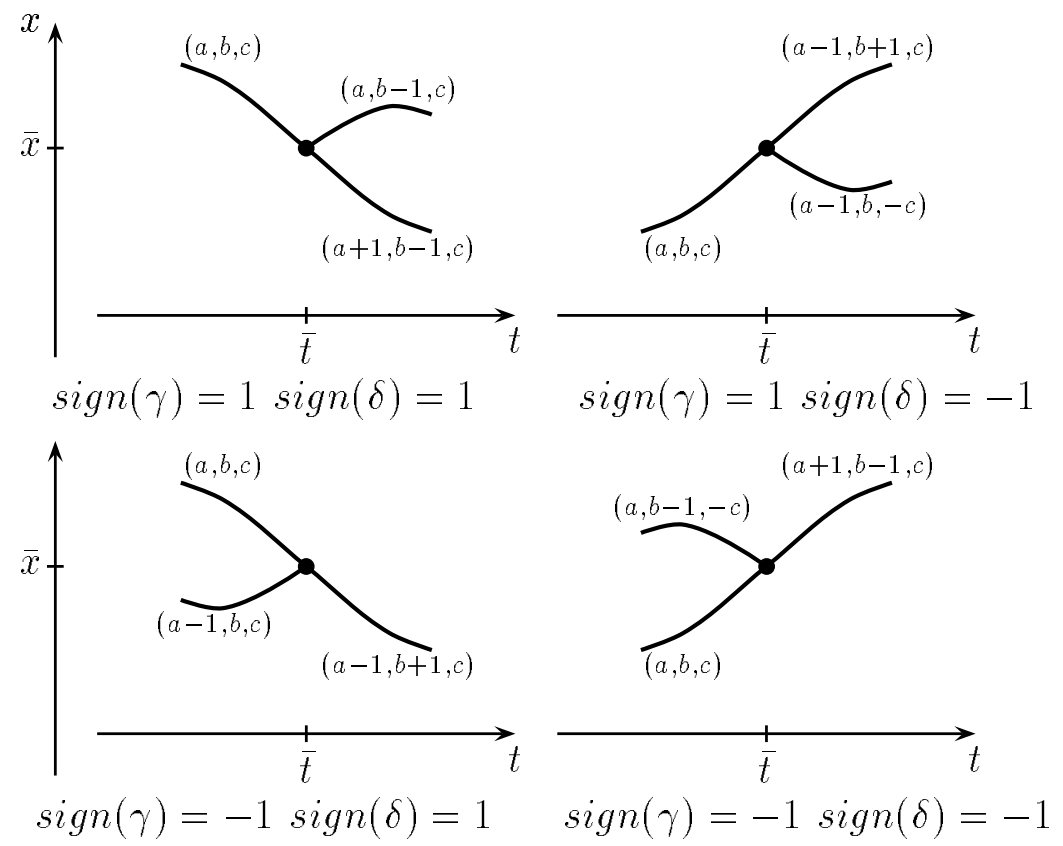

Figure 1: Type 2

Since $D_{x} g_{l}(\bar{z}) \cdot \dot{x}^{J_{0}}(\bar{t})+D_{t} g_{l}(\bar{z})=0$, it follows that

$$
\gamma=D_{x} g_{l}(\bar{z}) \cdot \dot{x}^{J_{0}^{+}}(\bar{t})+D_{t} g_{l}(\bar{z})=D_{x} g_{l}(\bar{z})\left(\dot{x}^{J_{0}^{+}}(\bar{t})-\dot{x}^{J_{0}}(\bar{t})\right) .
$$

Now (5) provides $\gamma=-\dot{\mu}_{l}^{J_{0}}(\bar{t}) \cdot D_{x} g_{l}(\bar{z}) V_{0}\left(V_{0}^{T} D_{x} \mathrm{~L} V_{0}\right)^{-1} V_{0}^{T} D_{x}^{T} g_{l}(\bar{z})$. Since $V_{0}=\left[V_{1} \vdots v_{1}\right]$, it follows that

$$
\gamma=-\dot{\mu}_{l}^{J_{0}}(\bar{t}) \cdot\left(0 \vdots D_{x} g_{l}(\bar{z}) v_{1}\right)^{T}\left(\begin{array}{cc}
V_{1}^{T} D_{x} \mathrm{~L} V_{1} & V_{1}^{T} D_{x} \mathrm{~L} v_{1} \\
v_{1}^{T} D_{x} \mathrm{~L} V_{1} & v_{1}^{T} D_{x} \mathrm{~L} v_{1}
\end{array}\right)^{-1}\left(\begin{array}{c}
0 \\
v_{1}^{T} D_{x}^{T} g_{l}(\bar{z})
\end{array}\right) .
$$

Lemma 1 and (4) together give:

$$
\gamma=-\dot{\mu}_{l}^{J_{0}}(\bar{t}) \cdot\left(D_{x} g_{l}(\bar{z}) v_{1}^{T}\right)^{2}\left(\left.D_{x} \mathrm{~L}\right|_{T_{\bar{x}}^{+} M(\bar{t})}\left|D_{x} \mathrm{~L}\right|_{T_{\bar{x}} M(\bar{t})}\right)^{-1} .
$$

Substituting (4) in the above relation concludes the proof. $\square$

Using Proposition 1 we obtain the changes of the triple (3) as shown in Figure 1.

Definition 4 (see also [11])

A g.c. point $\bar{z}$ of $V I(F, H, G)$ is of Type 3 if the following conditions hold: 
Type3: 1-VU : The LICQ is fulfilled.

Type3: 2-VU : VU-ND1 is fulfilled.

Type3: 3-VU : $\left.D_{x} \mathrm{~L}(\bar{z}, \bar{\lambda}, \bar{\mu})\right|_{T_{\bar{x}} M(\bar{t})}$ has rank $n-m-\left|J_{0}(\bar{z})\right|-1$.

Type3: 4-VU : Let us denote

- $\Pi=\left(H, G_{J_{0}(\bar{z})}\right)$,

- $W$ is a matrix whose columns form a linear basis of $T_{\bar{x}} M(\bar{t})$,

- $w_{1} \neq 0$ is a vector, with $W^{T} D_{x} \mathrm{~L}(\bar{z}, \bar{\lambda}, \bar{\mu}) W \cdot w_{1}=0, v_{1}=W \cdot w_{1}$, and

- $w_{2} \neq 0$ is a vector, with $W^{T} D_{x}^{T} \mathrm{~L}(\bar{z}, \bar{\lambda}, \bar{\mu}) W \cdot w_{2}=0, v_{2}=W \cdot w_{2}$.

It holds that $\beta_{1} \cdot \beta_{2} \neq 0$, where

$$
\begin{aligned}
\beta_{1}= & v_{1}^{T}\left(D_{x}^{2} \mathrm{~L} \cdot v_{2}\right) v_{1}-2 v_{1}^{T} D_{x}^{T} \mathrm{~L}\left(\left(D_{x}^{T} \Pi\right)^{\dagger}\right)^{T} \cdot\left(v_{1}^{T} D_{x}^{2} \Pi \cdot v_{2}\right) \\
& -v_{2}^{T} D_{x} \mathrm{~L}\left(\left(D_{x}^{T} \Pi\right)^{\dagger}\right)^{T}\left(v_{1}^{T} D_{x}^{2} \Pi v_{1}\right), \\
\beta_{2}= & D_{t} \mathrm{~L} \cdot v_{2}-D_{t}^{T} \Pi \cdot\left(D_{x}^{T} \Pi\right)^{\dagger} D_{x}^{T} \mathrm{~L} \cdot v_{2}
\end{aligned}
$$

and the following notation is used:

$$
\begin{array}{r}
v_{1}^{T}\left(D_{x}^{2} \mathrm{~L} \cdot v_{2}\right) v_{1}=v_{1}^{T}\left[D_{x}\left(D_{x}^{T} \mathrm{~L} \cdot v_{2}\right)\right] v_{1}^{T}, \\
v_{1}^{T} D_{x}^{2} \Pi v_{2}=\left(v_{1}^{T} D_{x}^{2} h_{i} v_{2}, i \in I, v_{1}^{T} D_{x}^{2} g_{j} v_{2}, j \in J_{0}\right)^{T} .
\end{array}
$$

Remark 4 (see also [16])

Under the LICQ and Type3: 3-VU the condition Type3: 4-VU is equivalent to the non-degeneracy of the vector $(\bar{z}, \bar{\lambda}, \bar{\mu})$ as a critical point of the optimization problem:

$$
\begin{aligned}
& \mathcal{P}_{V U} \min _{(z, \lambda, \mu) \in \mathcal{X}_{V U}} \Lambda(z, \lambda, \mu)=t, \\
& \quad \mathcal{X}_{V U}=\left\{(z, \lambda, \mu) \in \mathbb{R}^{n+m+\left|J_{0}(\bar{z})\right|+1} \mid \mathrm{H}_{J_{0}}(z, \lambda, \mu)=0\right\} .
\end{aligned}
$$

\section{Remark 5}

$\Sigma_{g c}$ is locally described by the projection of $\mathcal{X}_{V U}$ onto the z-components. Consequently, $\Sigma_{g c}$ can be parametrized around $\bar{z}$ by one of the $x$-components, and the local structure is as shown in Figure 2. 


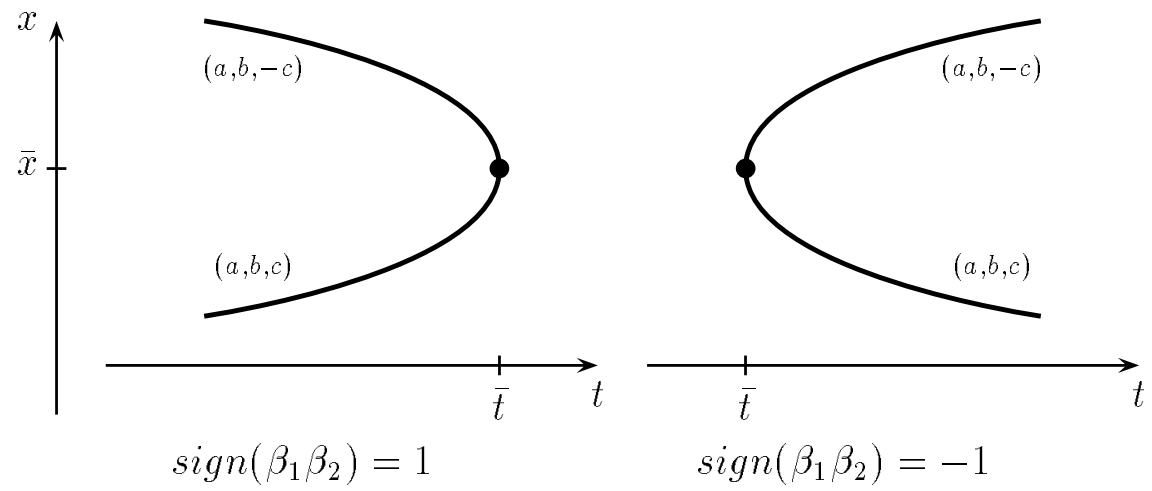

Figure 2: Type 3

In $\bar{z}$ the curve $\Sigma_{g c}$ has a turning point in the variable $t$, since $(\bar{z}, \bar{\lambda}, \bar{\mu})$ is a locally maximizer or minimizer of $\mathcal{P}_{V U}$. The position of the turning point $\bar{z}(t \geq \bar{t}$ as locally minimizer, the other $t \leq \bar{t}$ as locally maximizer $)$ depends directly on $\operatorname{sign}\left(\beta_{1} \beta_{2}\right)$ (see Figure 2 ). It is easy to note that $\operatorname{sign}\left(\beta_{1}\right)$ and $\operatorname{sign}\left(\beta_{1} \beta_{2}\right)$ are independent of the selected vectors $v_{1}$ and $v_{2}$ in Type3: 4VU.

The linear indices remain locally constant along $\Sigma_{g c}$. The local changes of sign $\left[\operatorname{det}\left(\left.D_{x} \mathrm{~L}(z, \lambda, \mu)\right|_{T_{x} M(t)}\right)\right]$ are explained in Theorem 1 and in Remark 17.

Definition 5 (see also [11])

A g.c. point $\bar{z}$ of $V I(F, H, G)$ is of Type \& if the following conditions hold:

Type4: 1-VU : $0<m+\left|J_{0}(\bar{z})\right|<n+1$.

Type4: 2-VU : The matrix $D_{x}\left[\begin{array}{c}H(\bar{z}) \\ G_{J_{0}(\bar{z})}(\bar{z})\end{array}\right]$ has rank $m+\left|J_{0}(\bar{z})\right|-1$.

Type4: 3-VU : Let $(\bar{\lambda}, \bar{\mu})$ satisfying $(\bar{\lambda}, \bar{\mu}) \cdot D_{x}\left[\begin{array}{c}H(\bar{z}) \\ G_{J_{0}(\bar{z})}(\bar{z})\end{array}\right]=0$ be fixed. Then it holds that $\mu_{j} \neq 0, \forall j \in J_{0}(\bar{z})$.

Type4: 4-VU : $D_{t} \mathbf{L}(\bar{z}) \neq 0$ and the matrix $A=D_{t} \mathbf{L}(\bar{z}) \cdot W^{T} D_{x}^{2} \mathbf{L}(\bar{z}) W$ is regular, where $\mathbf{L}(z)=\sum_{i \in I} \bar{\lambda}_{i} h_{i}(z)+\sum_{j \in J_{0}(\bar{z})} \bar{\mu}_{j} g_{j}(z)$ and the columns of $W$ form a basis for the $\left(n-m-J_{0}(\bar{z})+1\right)$-dimensional linear space

$$
T=\left\{\eta \in \mathbb{R}^{n} \mid D h_{i}(\bar{z}) \eta=0, D g_{j}(\bar{z}) \eta=0, i \in I, j \in J_{0}(\bar{z})\right\}
$$


Type4: 5-VU : $F^{T}(\bar{z}) W A^{-1} W^{T} F(\bar{z}) \neq 0$.

Let us fix $l \in J_{0}(\bar{z})$ and denote $\theta=\left(\theta_{0}, \theta_{i}, \theta_{j}, i \in I, j \in J_{0}(\bar{z}) \backslash\{l\}\right)$.

Remark 6 (see also [16])

If Type4: 1-VU, Type4: 2-VU and Type4: 3-VU are fulfilled, the conditions Type4: 4-VU and Type4: 5-VU together are equivalent to the following non-degeneracy:

The vector $(\bar{z}, \bar{\theta})$, where $\bar{\theta}=\left(0, \bar{\lambda}, \bar{\mu}_{j}, j \in J_{0}(\bar{z}) \backslash l\right)$, is a non-degenerated critical point of the problem $\widetilde{\mathcal{P}}_{V U}$ :

$$
\begin{array}{lll}
\widetilde{\mathcal{P}}_{V U} & \min & \tilde{\Lambda}(z, \theta)=t, \\
& (z, \theta) \in \widetilde{\mathcal{X}}_{V U} & \\
& \widetilde{\mathcal{X}}_{V U}=\left\{(z, \theta) \in \mathbb{R}^{n+m+\left|J_{0}(\bar{z})\right|+1} \mid \Gamma_{V U}(z, \theta)=0\right\}
\end{array}
$$

Here $\Gamma_{V U}(z, \theta)$ is defined by:

$$
\Gamma_{V U}(z, \theta)=\left(\begin{array}{c}
\theta_{0} F(z)-\sum_{i \in I} \theta_{i} D_{x} h_{i}(z)-\sum_{j \in J_{0}(\bar{z}) \backslash\{l\}} \theta_{j} D_{x} g_{j}(z)-\bar{\mu}_{l} D_{x} g_{l}(z) \\
H(z) \\
G_{J_{0}(\bar{z})}(z)
\end{array}\right)
$$

\section{Remark 7}

Since $W^{T} F(\bar{z}) \neq 0$, the vectors

$$
\left\{F(\bar{z}), D_{x} h_{i}(\bar{z}), D_{x} g_{j}(\bar{z}), i \in I, j \in J_{0}(\bar{z}) \backslash\{k\}\right\}
$$

are linearly independent for arbitrary $k \in J_{0}(\bar{z})$. Therefore, the projection of $\widetilde{\mathcal{X}}_{V U}$ onto the $z$-components describes the set $\Sigma_{g c}$ around $\bar{z}$. $\widetilde{\mathcal{X}}_{V U}$ can be parametrized with a smooth mapping $(z(\tau), \theta(\tau))$ defined around zero satisfying $(z(0), \theta(0))=(\bar{z}, \bar{\theta})$. From the LICQ it follows that $\dot{t}(0)=0 \neq \dot{\theta}_{0}(0)$. Consequently, LICQ is fulfilled around $\bar{z}$ at each point of $\Sigma_{g c}$ different from $\bar{z}$. The linear indices change their values for $\tau<0$ and $\tau>0$, since the Lagrange-multipliers are given by $\frac{1}{\theta_{0}(\tau)}\left(\theta_{i}(\tau), \theta_{j}(\tau), i \in I, j \in J_{0}(\bar{z}) \backslash\{l\}, \bar{\mu}_{l}\right)$.

Lemma 2 (see [12])

Let $A$ be a symmetric $(n \times n)$-matrix and $B$ an $(n \times m)$ - matrix with rank $\bar{m}$. If $C=\left(\begin{array}{cc}A & B \\ B^{T} & 0\end{array}\right)$, it follows $\operatorname{In}(C)=\operatorname{In}\left(\left.C\right|_{\operatorname{Kern}^{T}}\right)+\operatorname{In}(\bar{m}, \bar{m}, m-\bar{m})$. 
Here $\operatorname{Kern} B^{T}=\left\{\xi \in \mathbb{R}^{n} \mid B^{T} \cdot \xi=0\right\}$ and $\left.C\right|_{\text {Kern } B^{T}}$ denotes a matrix of the form $\tilde{B}^{T} C \tilde{B}$, where the columns of $\tilde{B}$ form a linear basis for $\operatorname{Kern} B^{T}$.

\section{Proposition 2}

Let $(z(\tau), \theta(\tau))$ parametrize the curve $\widetilde{\mathcal{X}}_{V U}$ around a g.c. point $\bar{z}$ of Type 4 with $(z(0), \theta(0))=(\bar{z}, \bar{\theta}))$. Then, for $\tau \neq 0, z(\tau)$ is a non-degenerated g.c. point. sign $\left[\operatorname{det}\left(\left.D_{x} \mathrm{~L}(z(\tau))\right|_{\left.T_{x(\tau)} M(t(\tau))\right)}\right.\right.$ is the same (or different) for $\tau>0$ and $\tau<0$ if and only if $n-m-J_{0}(\bar{z})$ is even (resp. odd).

\section{Proof:}

Let $l \in J_{0}(\bar{z})$ be fixed. We use the following notations:

$B_{0}(z)=D_{x}\left(\begin{array}{c}H(z) \\ G_{J_{0}(\bar{z}) \backslash\{l\}}(z) \\ F^{T}(z)\end{array}\right), B_{1}(z)=D_{x}\left(\begin{array}{c}H(z) \\ G_{J_{0}(\bar{z}) \backslash\{l\}}(z)\end{array}\right)$, and $\mathcal{L}(z, \theta)=\theta_{0} F(z)-\sum_{i \in I} \theta_{i} D_{x} h_{i}(z)-\sum_{j \in J_{0}(\bar{z}) \backslash\{l\}} \theta_{j} D_{x} g_{j}(z)-\bar{\mu}_{l} D_{x} g_{l}(z)$.

Let $W_{0}(z)$ be defined in a neighbourhood $U_{\bar{z}}$ of $\bar{z}$ such that its columns form a linear basis for $\operatorname{Kern} B_{0}(z)$. $W_{0}(z)$ can be choosen depending from $z$ as smooth as $B_{0}(z)$. Let the columns of a matrix $W_{1}=\left[W_{0}(\bar{z}) \vdots b_{0}\right]$ form a basis for $\operatorname{Kern} B_{1}(z)$.

Consider $\tau$ near zero with $z(\tau) \in U_{\bar{z}}$. The columns of $W_{0}(z(\tau))$ form then a basis for $T_{x(\tau)} M(t(\tau))$ and, since LICQ and VU-ND1 are fulfilled by Remark 7, the non-degeneracy of $z(\tau)$ holds if $W_{0}^{T}(z(\tau)) D_{x} \mathrm{~L}(z(\tau)) W_{0}(z(\tau))$ is regular, where $D_{x} \mathrm{~L}(z(\tau))=\frac{1}{\theta_{0}(\tau)} D_{x} \mathcal{L}(z(\tau), \theta(\tau))$. Consequently, it is sufficient to show the regularity of

$$
W_{0}^{T}(z(0)) D_{x} \mathcal{L}(z(0), \theta(0)) W_{0}(z(0))=-W_{0}^{T}(\bar{z}) D_{x}^{2} \mathbf{L}(\bar{z}) W_{0}(\bar{z}),
$$

where $\mathbf{L}(z)$ is intended as in Definition 5. The equation

$$
\operatorname{det}\left(W_{0}^{T}(z(\tau)) D_{x} \mathrm{~L}(z(\tau)) W_{0}(z(\tau))\right)=\left(\frac{1}{\theta_{0}(\tau)}\right)^{\left(n-m-J_{0}(\bar{z})\right)} \operatorname{det}\left(W_{0}^{T}(z(\tau)) D_{x} \mathcal{L}(z(\tau), \theta(\tau)) W_{0}(z(\tau))\right)
$$

explains the changes of $\operatorname{sign}\left[\operatorname{det}\left(\left.D_{x} \mathrm{~L}(z(\tau))\right|_{T_{x(\tau)} M(t(\tau))}\right)\right]$.

Using Lemma 2 we obtain that the matrix (7) is regular if and only if

$$
\left(\begin{array}{lll}
D_{x}^{2} \mathbf{L}(\bar{z}) & B_{1}(\bar{z}) & F(\bar{z}) \\
B_{1}^{T}(\bar{z}) & 0 & 0 \\
F^{T}(\bar{z}) & 0 & 0
\end{array}\right)
$$


is so, too, and that the submatrix $\left(\begin{array}{ll}D_{x}^{2} \mathbf{L}(\bar{z}) & B_{1}(\bar{z}) \\ B_{1}^{T}(\bar{z}) & 0\end{array}\right)$ is regular, since $A$ (as in Definition 5) is nonsingular. From Lemma 1 the regularity of (9) is obtained if

$$
\left(\begin{array}{ll}
F^{T}(\bar{z}) & 0
\end{array}\right)\left(\begin{array}{ll}
D_{x}^{2} \mathbf{L}(\bar{z}) & B_{1}(\bar{z}) \\
B_{1}^{T}(\bar{z}) & 0
\end{array}\right)^{-1}\left(\begin{array}{l}
F(\bar{z}) \\
0
\end{array}\right) \neq 0 .
$$

However the above number is equal to $F^{T}(\bar{z}) W_{1}\left[W_{1}^{T} D_{x}^{2} \mathbf{L}(\bar{z}) W_{1}\right]^{-1} W_{1}^{T} F(\bar{z})$, and the proof is concluded by using Type4: 5 -VU.

\section{Remark 8}

$\dot{x}(0) \neq 0$, since the vectors $(6)$ are linearly independent and $\dot{t}(0)=0 . \Sigma_{g c}$ is then parametrizable around $\bar{z}$ with respect to an $x$-component (see Figure 3 ).

Around $\bar{z} \Sigma_{g c}$ has a turning point in $t$, since $(\bar{z}, \bar{\theta})$ is a non-degenerated stationary point of $\tilde{\mathcal{P}}_{V U}$. The geometric position of the turning point is determined by the number

$$
\left.D_{(z, \theta)}^{2} L(\bar{z}, \bar{\theta})\right|_{T_{(\bar{z}, \bar{\theta})}} \tilde{\mathcal{X}}_{V U}=\frac{-1}{D_{t} \mathbf{L}(\bar{z})} \cdot F^{T}(\bar{z}) W\left[W^{T} D_{x}^{2} \mathbf{L}(\bar{z}) W\right]^{-1} W^{T} F(\bar{z}),
$$

where $L$ is the Lagrange-function corresponding to $\widetilde{\mathcal{P}}_{V U}$ and $W$ is intended as in Definition 5.

Since $\operatorname{sign}\left(\left.D_{(z, \theta)}^{2} L(\bar{z}, \bar{\theta})\right|_{T_{(\bar{z}, \bar{\theta})} \tilde{\mathcal{X}}_{V U}}\right)=-\operatorname{sign}\left(F^{T}(\bar{z}) W A^{-1} W^{T} F(\bar{z})\right)$, we can use $\alpha=\operatorname{sign}\left(F^{T}(\bar{z}) W A^{-1} W^{T} F(\bar{z})\right)$, for the characterization of the geometric position of the curve $\Sigma_{g c}$ (see Figure 3$)$.

Finally $\operatorname{sign}(\beta)=(-1)^{n-m-J_{0}(\bar{z})}$ determines according to Proposition 2 the changes of sign $\left[\operatorname{det}\left(\left.D_{x} \mathrm{~L}(z(\tau))\right|_{\left.T_{x(\tau)} M(t(\tau))\right)}\right)\right.$.

\section{Remark 9}

Let us consider a point $\bar{z}$ of Type 4 in the case that the sets $M(t)$ are convex $\left(h_{i}\right.$ linear affine and then $\left.J_{0}(\bar{z}) \neq \emptyset\right)$. Let $(z(\tau), \theta(\tau))$ be a parametrization as in the above Proposition $D^{2}$, and such that $z(\tau)$ is a stationary point for $\tau<0$. It follows that every $\bar{\mu}_{j}, j \in J_{0}(\bar{z})$, in Definition 5 has the same sign (i.e., the MFCQ is not fulfilled). Let us fix then $\bar{\mu}_{j}>0, \forall j \in J_{0}(\bar{z})$. It follows that $\theta_{0}(\tau)>0$ for $\tau<0$.

The LICQ holds for $\tau<0$ and the convexity of $M(t(\tau))$ implies that $D_{x}^{2} g_{j}(z(\tau)), j \in J_{0}(\bar{z})$, are negative semidefinite on $T_{x(\tau)} M(t(\tau))$. Therefore, 

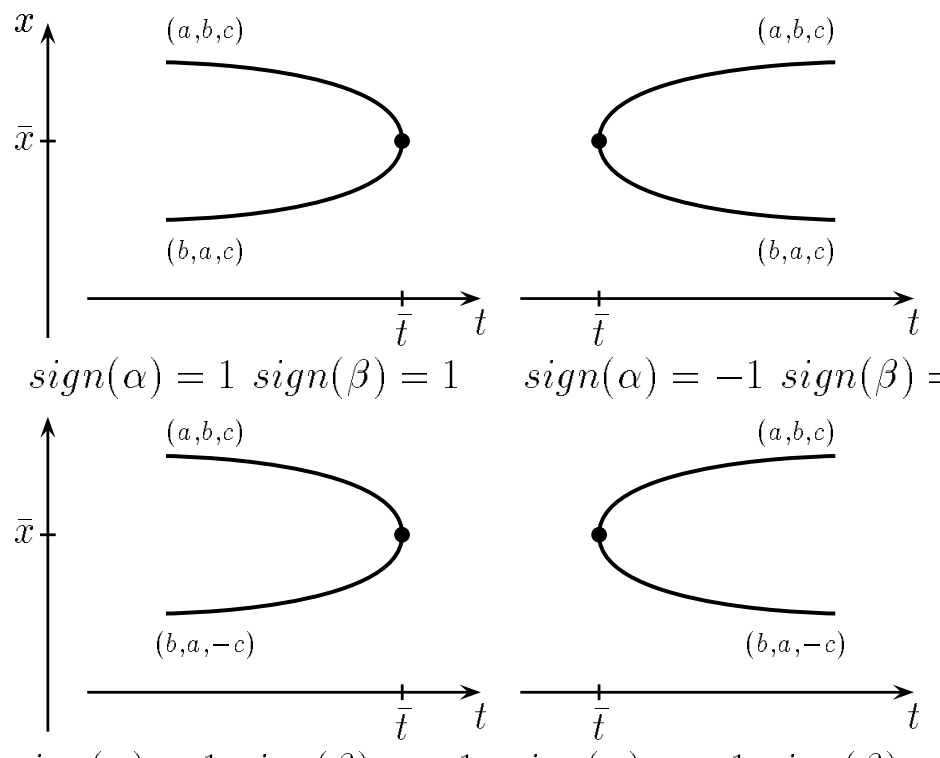

$\operatorname{sign}(\alpha)=-1 \operatorname{sign}(\beta)=1$

$\operatorname{sign}(\alpha)=1 \operatorname{sign}(\beta)=-1 \operatorname{sign}(\alpha)=-1 \operatorname{sign}(\beta)=-1$

Figure 3: Type 4

$W_{0}^{T} D_{x}^{2} \mathbf{L}(\bar{z}) W_{0}$ is negative semidefinite, where $W_{0}$ is intended as in the proof of the above Proposition 2 and $\mathbf{L}$ as in Definition 5. Since $W_{0}^{T} D_{x}^{2} \mathbf{L}(\bar{z}) W_{0}$ is regular, it is then negative definite, and sign $\left[\operatorname{det}\left(-W_{0}^{T} D_{x}^{2} \mathbf{L}(\bar{z}) W_{0}\right)\right]=+1$. The relations (7) and (8) imply then that sign $\left[\operatorname{det}\left(\left.D_{x} \mathrm{~L}(z(\tau))\right|_{\left.T_{x(\tau)} M(t(\tau))\right)}\right)\right]=$ +1 for $\tau<0$.

On the other hand, $W^{T} D_{x}^{2} \mathbf{L}(\bar{z}) W$, where $W$ is intended as in Definition 5, has at most one positive eigenvalue. Applying the results from Theorem 5.3 (cases 2. and 3.) in [10] about the homotopic changes of parametric feasible sets it follows that $W^{T} D_{x}^{2} \mathbf{L}(\bar{z}) W$ must be negative definite and that $M(t)$, by passing the parameter value $\bar{t}$, vanishes or is created.

Definition 6 (see also [11])

A g.c. point $\bar{z}$ of $V I(F, H, G)$ is of Type 5 if the following conditions hold:

Type5: 1-VU : $m+\left|J_{0}(\bar{z})\right|=n+1$.

Type5: 2-VU : The matrix $D\left[\begin{array}{c}H(\bar{z}) \\ G_{J_{0}(\bar{z})}(\bar{z})\end{array}\right]$ has rank $n+1$.

Type5: 3-VU : It holds Type4: 3-VU $((\bar{\lambda}, \bar{\mu})$ are fixed $)$. 
Type5: 4-VU : Let $(\bar{\alpha}, \bar{\beta}) \in \mathbb{R}^{m} \times \mathbb{R}^{\left|J_{0}(\bar{z})\right|}$ solve the system

$$
\left(\begin{array}{c}
F(\bar{z}) \\
0
\end{array}\right)-\sum_{i \in I} \bar{\alpha}_{i} D^{T} h_{i}(\bar{z})-\sum_{j \in J_{0}(\bar{z})} \bar{\beta}_{j} D^{T} g_{j}(\bar{z})=0 .
$$

Then $\Delta_{j k} \neq 0, \forall j, k \in J_{0}(\bar{z}), j \neq k$, where $\Delta_{j k}=\bar{\beta}_{j}-\bar{\beta}_{k} \cdot \bar{\mu}_{j}$.

\section{Remark 10}

From Type5: 1-VU, Type5: 2-VU and Type5: 3-VU it follows that each g.c. point in a neighbourhood of $\bar{z}$ solves (with corresponding multipliers) one of the systems

$$
\mathrm{H}_{J_{0}(\bar{z}) \backslash\{k\}}(z, \lambda, \mu)=0
$$

where $k \in J_{0}(\bar{z})$.

For each $k \in J_{0}(\bar{z})$ there exists a parametrization $\left(x^{k}(t), t, \lambda^{k}(t), \mu^{k}(t)\right)$ around $\left(\bar{z}, \bar{\lambda}^{k}, \bar{\mu}^{k}\right)$ of the solution set from $(10)$, with $\left(x^{k}(\bar{t}), \bar{t}, \lambda^{k}(\bar{t}), \mu^{k}(\bar{t})\right)=$ $\left(\bar{z}, \bar{\lambda}^{k}, \bar{\mu}^{k}\right)$.

\section{Remark 11}

$\Sigma_{g c}$ is described around $\bar{z}$ by the union of the feasible parts of the $n+1$ curves $\left(x^{k}(t), t\right)$. It also holds that $\frac{d}{d t} g_{k}\left(x^{k}(\bar{t}), \bar{t}\right)=D_{x}^{T} g_{k}(\bar{z}) \dot{x}^{k}(\bar{t})+D_{t} g_{k}(\bar{z}) \neq 0$ for $k \in J_{0}(\bar{z})$. Since $D_{x}^{T} g_{k_{1}}(\bar{z}) \dot{x}^{k_{2}}(\bar{t})+D_{t} g_{k_{1}}(\bar{z})=0$, it follows that $\dot{x}^{k_{1}}(\bar{t}) \neq \dot{x}^{k_{2}}(\bar{t})$ for $k_{1} \neq k_{2}$ (see Figure 4).

Remark 12 (see [11])

If the MFCQ fails to hold in $\bar{z}$, then every $\bar{\mu}_{k}$ has the same sign. Then each curve belonging to $\Sigma_{g c}$ runs in the same t-direction. Therefore, $\bar{z}$ is a turning point from $\Sigma_{g c}$. Analogously as for optimization problems it holds that $\Sigma_{\text {stat }}$ around $\bar{z}$ consists of only one curve $\left(x^{k}(t), t\right)$ and $\bar{z}$ is a border point of $\Sigma_{\text {stat }}$.

If the MFCQ is fulfilled and $\bar{z}$ is a stationary point, then there exist exactly two curves $\left(x^{k_{1}}(t), t\right)$ and $\left(x^{k_{2}}(t), t\right)$ consisting of stationary points and with opposite t-directions. Hence, $\Sigma_{\text {stat }}$ has a continuation.

The local structure of the sets $\Sigma_{g c}$ and $\Sigma_{\text {stat }}$ around a point of Type 5 is shown in figure 4 .

\section{Remark 13}

If the $M(t)$ are convex ( $h_{i}$ affine and $J_{0}(\bar{z}) \neq \emptyset$, since $|I|<=n$ ) Theorems 4.1 (Type 3 and 4) and 5.3 (cases 2. and 3.) in [10] imply that the feasible set $M(t)$ in the parameter value $\bar{t}$ vanishes or appears when the MFCQ is not fulfilled. 


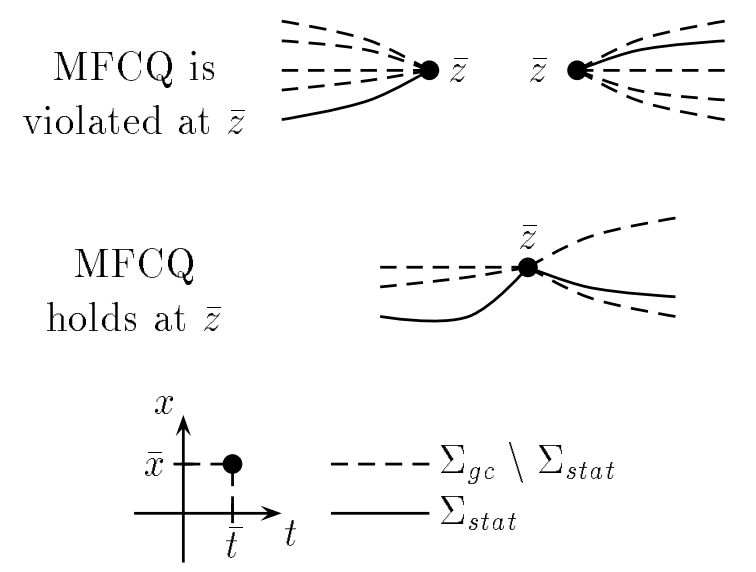

Figure 4: Typ 5

\section{Some properties of regular one-parametric variational inequalities.}

\subsection{The points of Type 3 .}

In this section we prove a basic result similar to Theorem 10.2.2 in [9]. Let $q \leq n$ be fixed and consider mappings with the structure

$$
T(x, u, t)=\left(\begin{array}{c}
T^{1}(x, u, t) \\
T^{2}(x, t)
\end{array}\right)
$$

where $x \in \mathbb{R}^{n}, u \in \mathbb{R}^{q}, T: \mathbb{R}^{n+q+1} \rightarrow \mathbb{R}^{n+q}, T^{1}: \mathbb{R}^{n+q+1} \rightarrow \mathbb{R}^{n}$ and therefore $T^{2}: \mathbb{R}^{n+1} \rightarrow \mathbb{R}^{q}$. Let us consider two matrices, $B_{L}=\left(\begin{array}{ll}C_{1} & C_{2} \\ 0 & I_{q}\end{array}\right)$ and $B_{R}=\left(\begin{array}{cc}C_{3} & 0 \\ C_{4} & I_{q}\end{array}\right)$, where $C_{1}$ and $C_{2}$ are quadratic regular matrices with size $n$, and $I_{q}$ states for the identity matrix of size $q . B_{L}$ and $B_{R}$ are then quadratic and regular.

By $T_{\left(B_{L}, B_{R}\right)}(y, v, t)=B_{L} \cdot T\left(B_{R} \cdot(y, v), t\right)=\left(\begin{array}{c}T_{\left(B_{L}, B_{R}\right)}^{1}(y, v, t) \\ T_{\left(B_{L}, B_{R}\right)}^{2}(y, t)\end{array}\right)$ let us denote another mapping. From the structure of $B_{L}$ and $B_{R}$ it follows that 
$T_{\left(B_{L}, B_{R}\right)}$ has the same dependence on its variables as $T$ (pointed out by the notations $T_{\left(B_{L}, B_{R}\right)}^{1}$ and $\left.T_{\left(B_{L}, B_{R}\right)}^{2}\right)$. It holds that $T(x, u, t)=0$ if and only if $T_{\left(B_{L}, B_{R}\right)}\left(B_{R}^{-1} \cdot(x, u), t\right)=0$. If we define $\Sigma(T)=\{(x, u, t) \mid T(x, u, t)=0\}$, it follows that

$$
\Sigma(T)=\left(\begin{array}{ll}
B_{R} & 0 \\
0 & 1
\end{array}\right) \Sigma\left(T_{\left(B_{L}, B_{R}\right)}\right) .
$$

Let us consider the following assumption for a mapping $T(x, u, t)$.

$$
P_{1}(T): \quad 0 \text { is a regular value of } T
$$

\section{Remark 14}

The relation

$$
D T_{\left.\left(B_{L}, B_{R}\right)\right)}(y, v, t)=B_{L} \cdot D T\left(B_{R} \cdot(y, v), t\right) \cdot\left(\begin{array}{ll}
B_{R} & 0 \\
0 & 1
\end{array}\right)
$$

implies that $P_{1}(T)$ holds if and only if $P_{1}\left(T_{\left(B_{L}, B_{R}\right)}\right)$ holds, too.

Let us define $\Sigma^{s}(T)=\left\{(x, u, t) \in \Sigma(T) \mid \operatorname{det}\left(D_{(x, u)} T(x, u, t)\right)=0\right\}$.

\section{Proposition 3}

Let $(x, u, t) \in \Sigma^{s}(T)$ and $(y, v, t) \in \Sigma^{s}\left(T_{\left(B_{L}, B_{R}\right)}\right)$ be fixed with $(x, u)=B_{R}$. $(y, v)$. Let $P_{1}(T)$ (and also $P_{1}\left(T_{\left(B_{L}, B_{R}\right)}\right)$ ) be fulfilled around $(x, u, t)$ (resp. $(y, v, t))$. Then, $(x, u, t)$ is a non-degenerated critical point of $\left.t\right|_{\Sigma(T)}$ if and only if $(y, v, t)$ is a non-degenerated critical point of $\left.t\right|_{\Sigma\left(T_{\left(B_{L}, B_{R}\right)}\right)}$.

Here $\left.t\right|_{\Sigma(T)}$ (analogously to $\left.\left.t\right|_{\Sigma\left(T_{\left(B_{L}, B_{R}\right)}\right)}\right)$ represents the optimization problem with the objective function $F(x, u, t)=t$ and the feasible set $\Sigma(T)$. Proposition 3 is proved easily writting down booth conditions.

\section{Remark 15}

Let $P_{1}(T)$ be fulfilled and $(y(\theta), v(\theta), t(\theta))$ be a local parametrization of the curve $\Sigma\left(T_{\left(B_{L}, B_{R}\right)}\right)$ around $(y, v, t) \in \Sigma^{s}\left(T_{\left(B_{L}, B_{R}\right)}\right)$, which is defined in a neighbourhood of zero and $(y(0), v(0), t(0))=(y, v, t)$. By (1D) the mapping

$$
\left(\begin{array}{l}
x(\theta) \\
u(\theta) \\
t(\theta)
\end{array}\right)=\left(\begin{array}{ll}
B_{R} & 0 \\
0 & 1
\end{array}\right)\left(\begin{array}{l}
y(\theta) \\
v(\theta) \\
t(\theta)
\end{array}\right)
$$

is also a local parametrization of $\Sigma(T)$ around $(x, u, t)$. 
Since $(x, u, t) \in \Sigma^{s}(T)$ and $(y, v, t) \in \Sigma^{s}\left(T_{\left(B_{L}, B_{R}\right)}\right)$, the mappings

$$
\Psi(\theta)=\operatorname{det}\left[D_{(x, u)} T(x(\theta), u(\theta), t(\theta))\right]
$$

and $\Psi_{\left(B_{L}, B_{R}\right)}(\theta)=\operatorname{det}\left[D_{(y, v)} T_{\left(B_{L}, B_{R}\right)}(y(\theta), v(\theta), t(\theta))\right]$ vanish at $\theta=0$. Further, it holds that $\Psi_{\left(B_{L}, B_{R}\right)}(\theta)=\operatorname{det}\left[B_{L}\right] \cdot \operatorname{det}\left[B_{R}\right] \cdot \Psi(\theta)$. Consequently, $\Psi^{\prime}(0)=0$ if and only if $\Psi_{\left(B_{L}, B_{R}\right)}^{\prime}(0)=0$.

\section{Definition 7}

Let $\mathbf{M}_{1}(n+q)$ be the set of quadratic matrices of size $n+q$ having the submatrix formed by the last $q$ rows and columns equal to zero. $\mathbf{M}_{1}(n+q)$ can be identified with $\mathbb{R}^{n(n+2 q)}$. Let us define the open set

$$
\mathbf{M}_{2}(n+q)=\left\{\left(\begin{array}{cc}
A & B \\
C & 0
\end{array}\right) \in \mathbf{M}_{1}(n+q) \mid \operatorname{rank}(B)=\operatorname{rank}(C)=q\right\}
$$

and the manifold $\mathbf{M}_{3}(n+q, k)=\left\{P \in \mathbf{M}_{2}(n+q) \mid \operatorname{rank}(P)=k\right\}$, where $2 q \leq k \leq n+q$.

Due to the special dependence (11) of $T$ and $T_{\left(B_{L}, B_{R}\right)}$ on their variables it holds that $D_{(x, u)} T, D_{(y, v)} T_{\left(B_{L}, B_{R}\right)} \in \mathbf{M}_{1}(n+q)$.

\section{Remark 16}

Let $\bar{P} \in \mathbf{M}_{3}(n+q, k)$ be fixed. Due to the structure of $\bar{P}$ there exist two index sets $\{n+1, \ldots, n+q\} \subset I_{1}, I_{2} \subset\{1, \ldots, n+q\}$ such that $\left|I_{1}\right|=\left|I_{2}\right|=k$ and that the submatrix given by the intersection of the rows $I_{1}$ with the columns $I_{2}$ (and denoted by $\bar{P}_{\left(I_{1}, I_{2}\right)}$ ) is regular.

Let us denote $I_{1}^{c}=\{1, \ldots, n+q\} \backslash I_{1}$ (analogously $I_{2}^{c}$ ) and, by $\bar{P}_{\left(I_{1}^{c}, I_{2}\right)}$, $\bar{P}_{\left(I_{1}, I_{2}^{c}\right)}, \bar{P}_{\left(I_{1}^{c}, I_{2}^{c}\right)}$ the other submatrices of $\bar{P}$. Choose a neighbourhood $U(\bar{P}) \subset$ $\mathbf{M}_{1}(n+q)$ of $\bar{P}$ such that $U(\bar{P}) \subset \mathbf{M}_{2}(n+q)$ holds and that the submatrix $P_{\left(I_{1}, I_{2}\right)}$ is regular $\forall P \in U(\bar{P})$.

In $U(\bar{P})$ the manifold $\mathbf{M}_{3}(n+q, k)$ can be described by the equalities

$$
P_{\left(I_{1}^{c}, I_{2}^{c}\right)}-P_{\left(I_{1}^{c}, I_{2}\right)}\left[P_{\left(I_{1}, I_{2}\right)}\right]^{-1} P_{\left(I_{1}, I_{2}^{c}\right)}=0 .
$$

The gradients of these mappings are linearly independent (the partial derivatives with respect to the components of the matrix $P_{\left(I_{1}^{c}, I_{2}^{c}\right)}$ form an identity matrix of size $\left.(n+q-k)^{2}\right)$. Therefore, the set $\mathbf{M}_{3}(n+q, k)$ is a differentiable manifold with codimension $(n+q-k)^{2}$. 
Let us introduce the following notation

$$
\begin{aligned}
& M_{3}^{-1}=M_{3}(n+q, n+q-1) \\
& \hat{M}_{3}^{-1}=\left\{0_{(n+q)}\right\} \times M_{3}^{-1} .
\end{aligned}
$$

Consider for $T$ as in (11) the following condition

$$
P_{2}(T): \quad\left(T, D_{(x, u)} T\right) 历 \hat{M}_{3}^{-1} .
$$

The structure of $B_{L}$ and $B_{R}$ implies that $\left(T(\bar{x}, \bar{u}, \bar{t}), D_{(x, u)} T(\bar{x}, \bar{u}, \bar{t})\right) \in$ $\hat{M}_{3}^{-1}$ if and only if $\left(T_{\left(B_{L}, B_{R}\right)}(\bar{y}, \bar{v}, \bar{t}), D_{(y, v)} T_{\left(B_{L}, B_{R}\right)}(\bar{y}, \bar{v}, \bar{t})\right) \in \hat{M}_{3}^{-1}$, where $(\bar{y}, \bar{v}, \bar{t})=\left(B_{R}^{-1} \cdot(\bar{x}, \bar{u}), \bar{t}\right)$.

\section{Proposition 4}

$\operatorname{Let}\left(T(\bar{x}, \bar{u}, \bar{t}), D_{(x, u)} T(\bar{x}, \bar{u}, \bar{t})\right)$ and $\left(T_{\left(B_{L}, B_{R}\right)}(\bar{y}, \bar{v}, \bar{t}), D_{(y, v)} T_{\left(B_{L}, B_{R}\right)}(\bar{y}, \bar{v}, \bar{t})\right)$ belong to $\hat{M}_{3}^{-1}$ with $(\bar{y}, \bar{v}, \bar{t})=\left(B_{R}^{-1} \cdot(\bar{x}, \bar{u}), \bar{t}\right) . P_{2}(T)$ holds in a neighbourhood of $\left((\bar{x}, \bar{u}, \bar{t}), 0_{(n+q)}, D_{(x, u)} T(\bar{x}, \bar{u}, \bar{t})\right)$ if and only if $P_{2}\left(T_{\left(B_{L}, B_{R}\right)}\right)$ holds around $\left((\bar{y}, \bar{v}, \bar{t}), 0_{(n+q)}, D_{(y, v)} T_{\left(B_{L}, B_{R}\right)}(\bar{y}, \bar{v}, \bar{t})\right)$.

\section{Proof:}

Let $z_{1} \in \mathbb{R}^{n+q}$ and $z_{2} \in \mathbf{M}_{1}(n+q)$ be two variables. Denote by $\Phi\left(z_{2}\right)$ a differentiable mapping whose zero-set describes $\mathbf{M}_{3}^{-1}$ around $D_{(x, u)} T(\bar{x}, \bar{u}, \bar{t})$. $P_{2}(T)$ around $\left((\bar{x}, \bar{u}, \bar{t}), 0_{(n+q)}, D_{(x, u)} T(\bar{x}, \bar{u}, \bar{t})\right)$ is equivalent to the following fact: at the zero $\left(\bar{x}, \bar{u}, \bar{t}, 0_{(n+q)}, D_{(x, u)} T(\bar{x}, \bar{u}, \bar{t})\right)$ the mapping

$$
\Omega_{1}\left(x, u, t, z_{1}, z_{2}\right)=\left(\begin{array}{c}
z_{1}-T(x, u, t) \\
z_{2}-D_{(x, u)} T(x, u, t) \\
z_{1} \\
\Phi\left(z_{2}\right)
\end{array}\right)
$$

has a Jacobian-matrix with linearly independent rows.

Consider the linear transformation of coordinates

$$
\left.\left(\begin{array}{c}
x \\
u
\end{array}\right)\right)=\left(\begin{array}{c}
B_{R}\left(\begin{array}{c}
y \\
v
\end{array}\right) \\
t \\
z_{1} \\
z_{2}
\end{array}\right) .
$$


Here $\gamma_{1} \in \mathbb{R}^{n+q}$ and $\gamma_{2} \in \mathbf{M}_{1}(n+q)$ hold. The mapping $\Lambda\left(\gamma_{2}\right)=B_{L}^{-1} \gamma_{2} B_{R}^{-1}$ is linear and regular. Since $B_{L}^{-1}$ and $B_{R}^{-1}$ preserve the same structure as $B_{L}$ and $B_{R}$, it holds that $\Lambda\left(\gamma_{2}\right) \in \mathbf{M}_{3}^{-1}$ if and only if $\gamma_{2} \in \mathbf{M}_{3}^{-1}$.

If $\bar{\gamma}_{2}=B_{L} D_{(x, u)} T(\bar{x}, \bar{u}, \bar{t}) B_{R} \in \mathbf{M}_{3}^{-1}$, there exists a neighbourhood of $\bar{\gamma}_{2}$ in $\mathbf{M}_{1}(n+q)$ such that the zero-set of the mapping $\Psi\left(\gamma_{2}\right)=\Phi\left(B_{L}^{-1} \gamma_{2} B_{R}^{-1}\right)$ describes $\mathbf{M}_{3}^{-1}$ around $\bar{\gamma}_{2}$. $\Psi\left(\gamma_{2}\right)$ is differentiable and regular at $\bar{\gamma}_{2}$. In the new coordinates $\Omega_{1}$ is given by:

$$
\Omega_{2}\left(y, v, t, \gamma_{1}, \gamma_{2}\right)=\left(\begin{array}{c}
B_{L}^{-1} \gamma_{1}-T\left(B_{R}(y, v), t\right) \\
B_{L}^{-1} \gamma_{2} B_{R}^{-1}-D_{(x, u)} T\left(B_{R}(y, v), t\right) \\
B_{L}^{-1} \gamma_{1} \\
\Phi\left(B_{L}^{-1} \gamma_{2} B_{R}^{-1}\right)
\end{array}\right)
$$

$\operatorname{At}\left(\bar{y}, \bar{v}, \bar{t}, 0_{(n+q)}, \bar{\gamma}_{2}\right)=\left(B_{R}^{-1}(\bar{x}, \bar{u}), \bar{t}, 0_{(n+q)}, B_{L} D_{(x, u)} T(\bar{x}, \bar{u}, \bar{t}) B_{R}\right)$, the rows of the Jacobian-matrix of $\Omega_{2}$ are also linearly independent. The set of zeros of $\Omega_{2}$ around $\left(\bar{y}, \bar{v}, \bar{t}, 0_{(n+q)}, \bar{\gamma}_{2}\right)$ is the same as the one corresponding to the following mapping $\Omega_{3}$ (obtained from $\Omega_{2}$ by a linear transformation in target space).

$$
\begin{aligned}
\Omega_{3}\left(y, v, t, \gamma_{1}, \gamma_{2}\right) & =\left(\begin{array}{c}
\gamma_{1}-B_{L} T\left(B_{R}(y, v), t\right) \\
\gamma_{2}-B_{L} D_{(x, u)} T\left(B_{R}(y, v), t\right) B_{R} \\
\gamma_{1} \\
\Psi\left(\gamma_{2}\right)
\end{array}\right) \\
& =\left(\begin{array}{c}
\gamma_{1}-T_{\left(B_{L}, B_{R}\right)}(y, v, t) \\
\gamma_{2}-D_{(y, v)} T_{\left(B_{L}, B_{R}\right)}(y, v, t) \\
\gamma_{1} \\
\Psi\left(\gamma_{2}\right)
\end{array}\right) .
\end{aligned}
$$

At $\left(\bar{y}, \bar{v}, \bar{t}, 0_{(n+q)}, \bar{\gamma}_{2}\right)=\left(\bar{y}, \bar{v}, \bar{t}, T_{\left(B_{L}, B_{R}\right)}(\bar{y}, \bar{v}, \bar{t}), D_{(y, v)} T_{\left(B_{L}, B_{R}\right)}(\bar{y}, \bar{v}, \bar{t})\right)$ the mapping $\Omega_{3}$ vanishes and its Jacobian-matrix has linearly independent rows at this point. Hence, $P_{2}\left(T_{\left(B_{L}, B_{R}\right)}\right)$ holds around $\left(\bar{y}, \bar{v}, \bar{t}, 0_{(n+q)}, \bar{\gamma}_{2}\right)$.

Theorem 1 (for gradient-mappings see Theorem 10.2.2 in [9])

Let $T$ be as in (11) and $T(\bar{x}, \bar{u}, \bar{t})=0$ such that:

- $D_{(x, u, t)} T(\bar{x}, \bar{u}, \bar{t})$ has rank $n+q$.

- $\left(T(\bar{x}, \bar{u}, \bar{t}), D_{(x, u)} T(\bar{x}, \bar{u}, \bar{t})\right) \in \hat{M}_{3}^{-1}$

Then the following three conditions are equivalent: 
1. $(\bar{x}, \bar{u}, \bar{t})$ is a non-degenerated critical point of $\left.t\right|_{\Sigma(T)}$.

D. Let $(x(\theta), u(\theta), t(\theta))$, where $(x(0), u(0), t(0))=(\bar{x}, \bar{u}, \bar{t})$, parametrize the curve $\Sigma(T)$ locally and $\Psi(\theta)$ be as in (14). Then $\Psi^{\prime}(0) \neq 0$.

3. $P_{2}(T)$ holds in a neighbourhood of $\left((\bar{x}, \bar{u}, \bar{t}), 0_{(n+q)}, D_{(x, u)} T(\bar{x}, \bar{u}, \bar{t})\right)$.

\section{Proof:}

From the assumptions on $(\bar{x}, \bar{u}, \bar{t})$ it follows that:

- $(\bar{x}, \bar{u}, \bar{t}) \in \Sigma^{s}(T)$ and then $\Psi(0)=0$.

- $(\bar{x}, \bar{u}, \bar{t})$ is a critical point of $\left.t\right|_{\Sigma(T)}$, where the LICQ is fulfilled.

Since $D_{(x, u)} T(\bar{x}, \bar{u}, \bar{t}) \in \mathbf{M}_{3}^{-1}$, two matrices $B_{L}$ and $B_{R}$ can be selected such that:

$$
B_{L} \cdot D_{(x, u)} T(\bar{x}, \bar{u}, \bar{t}) \cdot B_{R}=\left(\begin{array}{lll}
0 & 0_{n-1}^{T} & 0_{q}^{T} \\
0_{n-1} & A_{1} & B_{1} \\
0_{q} & B_{2}^{T} & 0
\end{array}\right)
$$

Here $0_{n-1}$ and $0_{q}$ are the zero vectors in $\mathbb{R}^{n}$ and $\mathbb{R}^{q}$ respectively, $A_{1}$ is a quadratic matrix of size $(n-1)$, and $B_{1}, B_{2}$ are matrices of size $(n-1) \times q$. The submatrix $\left(\begin{array}{ll}A_{1} & B_{1} \\ B_{2}^{T} & 0\end{array}\right)$ is regular.

Taking into account the Remarks 14 and 15 and the Propositions 3 and 4, it can be supposed w.l.o.g. that $D_{(x, u)} T(\bar{x}, \bar{u}, \bar{t})$ possesses the structure given in (15).

Let us denote by $T_{1}^{1}(x, u, t)$ the first component of the mapping $T^{1}$, which is part of $T$. From the full rank of $D_{(x, u, t)} T(\bar{x}, \bar{u}, \bar{t})$ it follows that $D_{t} T_{1}^{1}(\bar{x}, \bar{u}, \bar{t}) \neq 0$.

Let $(\bar{x}, \bar{u}, \bar{t})$ be a non-degenerated critical point of $\left.t\right|_{\Sigma(T)}$. The corresponding multiplier-vector is $\frac{1}{D_{t} T_{1}^{1}(\bar{x}, \bar{u}, \bar{t})}\left(1,0_{n+q-1}\right)$. On the other hand, the tangent space $T_{(\bar{x}, \bar{u}, \bar{t})} \Sigma(T)$ is generated by the vector $\left(1,0_{n+q}\right)$. The second order condition for the non-degeneracy of $(\bar{x}, \bar{u}, \bar{t})$ with respect to $\left.t\right|_{\Sigma(T)}$ is then equivalent to the condition

$$
D_{x_{1}}^{2} T_{1}^{1}(\bar{x}, \bar{u}, \bar{t}) \neq 0 \text {. }
$$

The rest consists in proving that the other two conditions are equivalent to $(16)$. 
Let $(x(\theta), u(\theta), t(\theta))$ and $\Psi(\theta)$ be as in the second condition. Given two indices $k, l \in\{1, \ldots, n+q\}$, denote by $\Psi_{k, l}(\theta)$ the determinant of the $(k, l)$ minor of the matrix $D_{(x, u)} T(x(\theta), u(\theta), t(\theta))$. Then it holds:

$$
\Psi(\theta)=\sum_{j=1}^{n}(-1)^{j+1} D_{x_{j}} T_{1}^{1}(\theta) \cdot \Psi_{1, j}(\theta)+\sum_{k=1}^{q}(-1)^{n+k+1} D_{u_{k}} T_{1}^{1}(\theta) \cdot \Psi_{1, n+k}(\theta) .
$$

From (15) it follows:

$$
\begin{aligned}
\Psi_{1,1}(0) & \neq 0 \\
\Psi_{1, j}(0) & =0, \forall j=2, \ldots, n+q \\
D_{x_{j}} T_{1}^{1}(\bar{x}, \bar{u}, \bar{t}) & =0, \forall j=1, \ldots, n \\
D_{u_{k}} T_{1}^{1}(\bar{x}, \bar{u}, \bar{t}) & =0, \forall k=1, \ldots, q
\end{aligned}
$$

Moreover,

$$
\begin{aligned}
\Psi^{\prime}(0) & =\frac{d}{d \theta}\left[D_{x_{1}} T_{1}^{1}(x(0), u(0), t(0))\right] \cdot \Psi_{1,1}(0) \\
& =D_{(x, u, t)} D_{x_{1}} T_{1}^{1}(\bar{x}, \bar{u}, \bar{t}) \cdot\left(\begin{array}{c}
\dot{x}(0) \\
\dot{u}(0) \\
\dot{t}(0)
\end{array}\right) \cdot \Psi_{1,1}(0) .
\end{aligned}
$$

Since $(\dot{x}(0), \dot{u}(0), \dot{t}(0))$ belongs to $T_{(\bar{x}, \bar{u}, \bar{t}} \Sigma(T)$, it follows that $\Psi^{\prime}(0)=$ $D_{x_{1}}^{2} T_{1}^{1}(\bar{x}, \bar{u}, \bar{t}) \cdot \dot{x}_{1}(0) \cdot \Psi_{1,1}(0)$, where $\dot{x}_{1}(0) \neq 0$ and $\Psi_{1,1}(0) \neq 0$. Now it is obvious that the second condition is equivalent to (16).

Let $z \in \mathbb{R}^{n+q}$ and $\gamma \in M_{2}(n+q)$. (15) implies that $M_{3}^{-1}$ can be described around $D_{(x, u)} T(\bar{x}, \bar{u}, \bar{t})$ by the zeros of $\Lambda_{4}(\gamma)=\gamma_{1,1}-\tilde{\Lambda}_{4}(\gamma)$. Then $D_{\gamma} \tilde{\Lambda}_{4}\left(D_{(x, u)} T(\bar{x}, \bar{u}, \bar{t})\right)=0$ holds, too (see remark 16$)$.

The third condition is then equivalent to the fact that the Jacobian-matrix of

$$
\Lambda=\left(\begin{array}{c}
\Lambda_{1} \\
\Lambda_{2} \\
\Lambda_{3} \\
\Lambda_{4}
\end{array}\right)=\left(\begin{array}{c}
z-T(x, u, t) \\
\gamma-D_{(x, u)} T(x, u, t) \\
z \\
\gamma_{1,1}-\tilde{\Lambda}_{4}(\gamma)
\end{array}\right)
$$

has full rank at $(\bar{x}, \bar{u}, \bar{t}, \bar{z}, \bar{\gamma})=\left(\bar{x}, \bar{u}, \bar{t}, 0_{n+q}, D_{(x, u)} T(\bar{x}, \bar{u}, \bar{t})\right)$. 
This Jacobian-matrix has the following structure

$\left.\begin{array}{ccccc}D_{z} & D_{x_{1}} & D_{(x, u, t) \backslash\left\{x_{1}\right\}} & D_{\gamma_{1,1}} & D_{\gamma \backslash\left\{\gamma_{1,1}\right\}} \\ \Lambda_{1} & \Lambda_{2} \\ \Lambda_{2} & 0_{n+q} & D_{(x, u, t) \backslash\left\{x_{1}\right\}} T(\bar{x}, \bar{u}, \bar{t}) & 0 & 0 \\ \Lambda_{3} & -D_{x_{1}}^{2} T_{1}^{1}(\bar{x}, \bar{u}, \bar{t}) & \otimes & 1 & 0_{n(n+2 q)-1}^{T} \\ \Lambda_{4} & \otimes & \otimes & 0_{n(n+2 q)-1} & I_{n(n+2 q)-1} \\ I_{n+q} & 0 & 0 & 0 & 0 \\ 0 & 0 & 0 & 1 & 0_{n(n+2 q)-1}^{T}\end{array}\right]$

where $0_{k}$ represents the $k$-dimensional zero vector (as column), and $\otimes$ a submatrix not written in detail. $D_{(x, u, t) \backslash\left\{x_{1}\right\}} T(\bar{x}, \bar{u}, \bar{t})$ is regular, and a simple consideration of the above matrix provides the equivalence of its regularity (by rows) with (16).

\section{Remark 17}

From Remark \& it is known that the first condition of the above Theorem 1 is fulfilled at the points of Type 3. Here $T(x, u, t)=\mathrm{H}_{J_{0}}(z, u)$ with $u=(\lambda, \mu)$, $z=(x, t)$ and

$$
T_{2}(z)=\left[\begin{array}{c}
H(z) \\
G_{J_{0}}(z)
\end{array}\right]
$$

Let $(z(\theta), u(\theta))$ be a local parametrization of the set $\left\{\mathrm{H}_{J_{0}}=0\right\}$ around $(\bar{z}, \bar{\lambda}, \bar{\mu})=(z(0), u(0))$. We use the notations $D_{x} \mathrm{~L}(\theta)=D_{x} \mathrm{~L}(z(\theta), u(\theta))$ and $D_{x} T_{2}(\theta)=D_{x} T_{2}(z(\theta))$. Then the sign of

$$
\Psi(\theta)=\operatorname{det}\left[\begin{array}{ll}
D_{x} \mathrm{~L}(\theta) & -D_{x}^{T} T_{2}(\theta) \\
D_{x} T_{2}(\theta) & 0
\end{array}\right]
$$

is different for $\theta<0$ and $\theta>0$. Let $W(\theta)$ be matrices deppending smoothly from $\theta$, whose columns form for $\theta$ around zero a basis of $T_{\bar{x}(\theta)} M(t(\theta))$. The equation

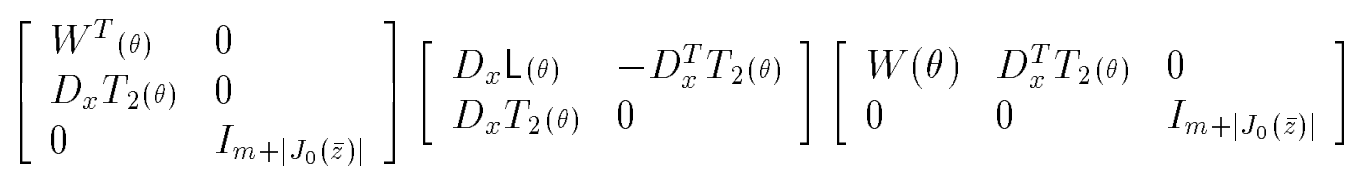




$$
=\left[\begin{array}{lll}
W^{T}(\theta) D_{x} \mathrm{~L}(\theta) W(\theta) & W^{T}(\theta) D_{x} \mathrm{~L}(\theta) D_{x}^{T} T_{2}(\theta) & 0 \\
D_{x} T_{2}(\theta) D_{x} \mathrm{~L}(\theta) W(\theta) & D_{x} T_{2}(\theta) D_{x} \mathrm{~L}(\theta) D_{x}^{T} T_{2}(\theta) & -D_{x} T_{2}(\theta) D_{x}^{T} T_{2}(\theta) \\
0 & D_{x} T_{2}(\theta) D_{x}^{T} T_{2}(\theta) & 0
\end{array}\right]
$$

implies the relation

$\Psi(\theta)\left(\operatorname{det}\left[\begin{array}{ll}W(\theta) & D_{x}^{T} T_{2}(\theta)\end{array}\right]\right)^{2}=\operatorname{det}\left[W^{T}(\theta) D_{x} \mathrm{~L}(\theta) W(\theta)\right]\left(\operatorname{det}\left[D_{x} T_{2}(\theta) D_{x}^{T} T_{2}(\theta)\right]\right)^{2}$

This fact explains the changes of $\operatorname{det}\left(\left.D_{x} \mathrm{~L}(z(\theta), u(\theta))\right|_{T_{\bar{x}(\theta)} M(t(\theta))}\right)$ over the curve $\Sigma_{g c}$ around the points of Type 3.

\subsection{Genericity and other results.}

\section{Definition 8}

Given $V I(F, H, G)$ define for $i=1, \ldots, 5$ the sets:

$$
\Sigma_{g c}^{i}(F, H, G)=\left\{z \in \Sigma_{g c}(F, H, G) \mid z \text { is of Type } i\right\} .
$$

We also use the notation $\Sigma_{g c}^{i}$.

Let us denote

$$
\mathcal{D}=C^{2}\left(\mathbb{R}^{n+1}, \mathbb{R}^{n}\right) \times C^{3}\left(\mathbb{R}^{n+1}, \mathbb{R}^{m+p}\right)
$$

and then define the set

$$
\mathcal{V}=\left\{(F, H, G) \in \mathcal{D} \mid \Sigma_{g c}=\cup_{i=1}^{5} \Sigma_{g c}^{i}\right\}
$$

\section{Definition 9}

A one-parametric variational inequality $(F, H, G) \in \mathcal{D}$ belonging to $\mathcal{V}$ will be called regular (in the sense of Jongen, Jonker and Twilt).

An important property of the regularity is its openness and its density with respect to the strong Whitney topology (see e.g. [1], [7], [9]). Let us state the local openness in the following.

\section{Theorem 2}

$\operatorname{Let}(\bar{F}, \bar{H}, \bar{G}) \in \mathcal{D}$ and $\bar{z} \in \Sigma_{g c}^{i}(\bar{F}, \bar{H}, \bar{G})$ (or $\left.\bar{z} \notin \Sigma_{g c}(\bar{F}, \bar{H}, \bar{G})\right), i \in\{1, \ldots, 5\}$. 
Then there exist an open neighbourhood $U_{\bar{z}}$ from $\bar{z}$ and a positive number $r_{\bar{z}}$ such that $\Sigma_{g c}(\tilde{F}, \tilde{H}, \tilde{G}) \cap U_{\bar{z}} \subset \Sigma_{g c}^{i}(\tilde{F}, \tilde{H}, \tilde{G}) \cup \Sigma_{g c}^{1}(\tilde{F}, \tilde{H}, \tilde{G})$ (resp. $\left.\Sigma_{g c}(\tilde{F}, \tilde{H}, \tilde{G}) \cap U_{\bar{z}}=\emptyset\right)$ holds $\forall(\tilde{F}, \tilde{H}, \tilde{G})$ such that

$$
\begin{array}{r}
\sup _{z \in U_{\bar{z}}}\left\{\max _{\alpha \in\{0,1,2\}}\left|D^{\alpha}(\tilde{F}-\bar{F})(z)\right|\right\}<r_{\bar{z}} \\
\sup _{z \in U_{\bar{z}}}\left\{\max _{\alpha \in\{0,1,2,3\}}\left|D^{\alpha}\left(\left[\begin{array}{c}
\tilde{H} \\
\tilde{G}
\end{array}\right]-\left[\begin{array}{c}
\bar{H} \\
\bar{G}
\end{array}\right]\right)(z)\right|\right\}<r_{\bar{z}} .
\end{array}
$$

This Theorem can be proved by continuity arguments taken into account the definition of the 5 singularities.

For the next perturbation theorem we fix the following notations. Let $V I(\bar{F}, \bar{H}, \bar{G})$ be fixed. Let us identify the space of every $k \times l$ matrix with $\mathbb{R}^{k l}$ and consider the following parameters: $A \in \mathbb{R}^{n^{2}}$ a quadratic matrix, $b \in \mathbb{R}^{n}$ $a$ column vector, $C \in \mathbb{R}^{(m+p) n}$ a matrix with $m+q$ rows and $n$ columns, and $d \in \mathbb{R}^{m+q}$ a column vector. Since only $A$ and $b$ are used for the perturbation of $F$, and $C$ and $d$ for the perturbation of $(H, G)$, we consider two parameter vectors $\mathcal{A}=(A, b)$ and $\mathcal{C}=(C, d)$. As a vector containing all perturbation parameters we use the notation $\mathcal{P}=(\mathcal{A}, \mathcal{C}) \in \mathbb{R}^{n^{2}+n+n(m+p)+m+p}$.

For a parameter value $\mathcal{P}$ we denote by

$$
(\bar{F}, \bar{H}, \bar{G}, \mathcal{P})=(\bar{F}(x, t)+A x+b,(\bar{H}(x, t), \bar{G}(x, t))+C x+d)
$$

the resulting perturbed one-parametric variational inequality. $(\bar{H}, \bar{G}, \mathcal{C})$, $(\bar{H}, \mathcal{C})_{i}, i \in I$ and $(\bar{G}, \mathcal{C})_{j}, j \in J$, represent the perturbed restrictions and their components. Analogously $(\bar{F}, \mathcal{A})$ denotes the perturbation of $\bar{F}$.

Theorem 3 (see also [16])

Let $\operatorname{VI}(\bar{F}, \bar{H}, \bar{G})$ be fixed with smooth data. Each measurable subset of

$$
\left\{\mathcal{P} \in \mathbb{R}^{n^{2}+n+n(m+p)+m+p} \mid(\bar{F}, \bar{H}, \bar{G}, \mathcal{P}) \notin \mathcal{V}\right\}
$$

has the Lebesgue measure zero.

The proof follows the same lines as the corresponding result for the class $\mathcal{F}$ in [16]. We give here only the main ideas of the extensive proof in [16] and indicate where new arguments are needed for our case.

\section{Proof:}


Lemma 1 in [16] can be used in our case without any changes. The considerations around the points of Type 4 and 5 in the second step also apply without major differences. Let us only note that for almost all $\mathcal{A}=$ $(A, b) \in \mathbb{R}^{n^{2}+n}$ (with non symmetric $A$ ) the following holds

$$
\begin{aligned}
F(\bar{x})+A \bar{x}+b & \notin T \\
(F(\bar{x})+A \bar{x}+b)^{T} Q(F(\bar{x})+A \bar{x}+b) & \neq 0,
\end{aligned}
$$

where $Q$ is a fixed nonvanishing $n \times n$-matrix, $T \subset \mathbb{R}^{n}$ is a fixed proper linear subspace and $\bar{x}, F(\bar{x}) \in \mathbb{R}^{n}$ are two fixed vectors.

For the first step consider two fixed index sets $J_{1} \subset J_{0} \subset\{1, \ldots, p\}$ with $0 \leq q=m+\left|J_{0}\right| \leq n$ and two numbers $\eta$ and $s$ with $\eta \in\{0,1\}$, respectively, $0 \leq 2(q+\eta-1) \leq s \leq n+q+\eta-1$. In the variables $\left(x, \lambda, \mu, t, \gamma_{1}, \gamma_{2}\right)$, where $\mu \in \mathbb{R}^{J_{0}}, \gamma_{1} \in \mathbb{R}^{n}$ and $\gamma_{2} \in M_{2}(n+q+\eta-1)$, consider the manifold

$M_{4}^{\eta}=\mathbb{R}^{n} \times \mathbb{R}^{m} \times\left\{\mu \in \mathbb{R}^{J_{0}} \mid \mu_{j}=0, j \in J_{1}\right\} \times\left\{0_{n+q}\right\} \times M_{3}(n+q+\eta-1, s)$, which has codimension $\left|J_{1}\right|+n+q+(n+q+\eta-1-s)^{2}$.

Let us fix an index in $J_{0}$ (w.l.o.g. 1 ) and define the mappings

$$
\begin{aligned}
& \mathrm{L}_{J_{0}}^{\eta}(\mathcal{P}, x, \lambda, \mu, t)=(\bar{F}, \mathcal{A})-\sum_{i=1}^{m} \lambda_{i} D_{x}^{T}(\bar{H}, \mathcal{C})_{i}-\eta \mu_{1} D_{x}^{T}(\bar{G}, \mathcal{C})_{1} \\
& -\sum_{j \in J_{0} \backslash\{1\}} \mu_{j} D_{x}^{T}(\bar{G}, \mathcal{C})_{j} \\
& \mathbf{H}_{J_{0}}^{\eta}(\mathcal{P}, x, \lambda, \mu, t)=\left[\begin{array}{c}
\mathrm{L}_{J_{0}}^{\eta}(\mathcal{P}, x, \lambda, \mu, t) \\
(\bar{H}, \mathcal{C}) \\
(1-\eta) \mu_{1}+(\bar{G}, \mathcal{C})_{1} \\
(\bar{G}, \mathcal{C})_{J_{0} \backslash\{1\}}
\end{array}\right] \\
& \mathrm{M}^{1}{ }^{\mathcal{P}, x, \lambda, \mu, t)}=D_{(x, \lambda, \mu)} \mathrm{H}_{J_{0}}^{1}(\mathcal{P}, x, \lambda, \mu, t) \in M_{1}(n+q) \\
& \mathbf{M}^{0}(\mathcal{P}, x, \lambda, \mu, t)=\left[\begin{array}{lll}
D_{x} \mathrm{~L}_{J_{0}}^{1} & D_{\lambda} \mathrm{L}_{J_{0}}^{0} & D_{\mu \backslash\left\{\mu_{1}\right\}} \mathrm{L}_{J_{0}}^{0} \\
D_{x}(\bar{H}, \mathcal{C}) & 0 & 0 \\
D_{x}(\bar{G}, \mathcal{C})_{J_{0} \backslash\{1\}} & 0 & 0
\end{array}\right] \in M_{1}(n+q-1)
\end{aligned}
$$

It can be shown that $\operatorname{Graf}\left(\mathrm{H}_{J_{0}}^{\eta}, \mathbf{M}^{\eta}\right) 历 \mathbb{R}^{n^{2}+n+n(m+p)+m+p} \times M_{4}^{\eta}$ for each possible selection of $J_{1}, J_{0}, \eta, s$ and of the fixed index. By use of the wellknown parametrized theorem of Sard it follows for almost all fixed $\mathcal{P}$ that, for each of the possible selections above, it holds that

$$
\operatorname{Graf}\left(\mathrm{H}_{J_{0}}^{\eta}, \mathrm{M}^{\eta}\right) 历 M_{4}^{\eta} .
$$


The codimension of $\operatorname{Graf}\left(\mathrm{H}_{J_{0}}^{\eta}, \mathrm{M}^{\eta}\right)$ is $n+q+n(n+2 q)$. If (17) is not the empty set, then $\left|J_{1}\right|+(n+q+\eta-1-s)^{2} \leq 1$.

For each g.c. point $\bar{z}$ of the problem $(\bar{F}, \bar{H}, \bar{G}, \mathcal{P})(\mathcal{P}$ is already fixed and (17) holds), where LICQ holds, there exists an active index set $J_{0}=J_{0}(\bar{z})$, multipliers $(\bar{\lambda}, \bar{\mu})$ and $J_{1}=\left\{j \in J_{0} \mid \bar{\mu}_{j}=0\right\}$ such that (17) holds for $\eta=1$.

If $s=n+q-1$ it follows $J_{1}=\emptyset$ (VU-ND1) and due to the definition of $M_{3}(n+q, n+q-1)$ it holds also Type3: 3-VU. Now (17) implies that $D_{(z, \lambda, \mu)} \mathrm{H}_{J_{0}}^{1}(\mathcal{P}, \bar{z}, \bar{\lambda}, \bar{\mu})$ has full rank. From Theorem 1 and Remark 4 we know that Type3: 4-VU holds and then $\bar{z}$ is of Type 3. In case $s=n+q$ the same arguments as in [16] lead to points of Type 1 or 2.

\section{Theorem 4}

The set $\mathcal{V}$ is open and dense in $\mathcal{D}$.

Here $\mathcal{D}$ is endowed with the product topology obtained by considering the $C^{2}$-strong (or Whitney) topology over $C^{2}\left(\mathbb{R}^{n+1}, \mathbb{R}^{n}\right)$ and the $C^{3}$-strong topology over $C^{3}\left(\mathbb{R}^{n+1}, \mathbb{R}^{m+p}\right)$.

\section{Proof:}

The proof follows from the Theorems 2 and 3 by use of the partition of the unity. The needed local $\rightarrow$ global construction is standard in the differential topology (see e.g. [1], [9]).

The following remarks about the structure of the critical curves hold with basically the same proof as in the case of one-parametric optimization problems (see e.g. [11]).

\section{Remark 18}

Let $V I(F, H, G) \in \mathcal{V}$. Remarks 1 and 2 imply the openness of the set $\Sigma_{g c}^{1}$ in $\Sigma_{g c}$. On the other hand, from Remark 3 (for Type 2), Remark 5 (for Type 3), the Remarks 7 and 8, Proposition 2(for Type 4) and the Remarks 10 and 11 (for Type 5), it follows that every point in $\Sigma_{g c} \backslash \Sigma_{g c}^{1}$ is isolated and a border points of $\Sigma_{g c}^{1}$. Therefore:

- $\Sigma_{g c}^{1}$ is open and dense in $\Sigma_{g c}$,

- $\Sigma_{g c} \backslash \Sigma_{g c}^{1}=\cup_{i=2}^{5} \Sigma_{g c}^{i}$ is a discrete set.

\section{Remark 19}

Let $V I(F, H, G) \in \mathcal{V}$. Remark Dimplies the openness of $\Sigma_{\text {stat }}^{1}$ in $\Sigma_{\text {stat. }}$. The changes of the linear indices around points of Type 2 and 3, shown in the figures 1 and 2 , imply that such points are not border points of the set $\Sigma_{\text {stat }}$. 
By definition the points of Type 4 do not belong to the set $\Sigma_{\text {stat }}$. If $z \in \Sigma_{g c}^{4}$ lies in $\overline{\Sigma_{\text {stat }}}$, then the MFCQ is violated and $\overline{\Sigma_{\text {stat }}}$ form a one-dimensional manifold (possibly with border) around $z$. In this case $z$ is a border point of $\overline{\Sigma_{\text {stat }}}$ if and only if $J_{0}(z) \neq \emptyset$. On the other hand, it follows from Remark 1 D that points of Type 5 in $\Sigma_{\text {stat }}$ are border points of $\Sigma_{\text {stat }}$ if and only if the MFCQ fails to hold.

\section{Acknowledgement}

I would like to thank Prof. Dr. H.Th. Jongen for his valuable comments and Prof. Dr. Jürgen Guddat for his support.

\section{References}

[1] Milnor J.: Topology from a differentiable viewpoint, University of Virginia Press, 1965.

[2] Facchinei F. und J. Soares: A new merit function for nonlinear complementarity problems and a related algorithm, SIAM J. Optim, Vol. 7, No. 1, (1997), 225-247.

[3] Fukushima M. und N. Yamashita: Equivalent unconstrained minimization and global error bounds for variational inequality problems, SIAM J. Control Optim., Vol. 35, No. 1, (1997) 273-284.

[4] Guddat J., F. Guerra und H.Th. Jongen: Parametric Optimization: singularities, pathfollowing and jumps. John Wiley, Chichester and Teubner, Stuttgart 1990.

[5] Harker P.T. und Jong-Shi Pang: Finite-dimensional variational inequality and nonlinear complementarity problems: A survey of theory, algorithms and applications. Mathematical Programming 48 (1990) 161-220.

[6] Harker P.T. und B. Xiao: A nonsmooth Newton method for variational inequalities, I: theory, Mathematical Programming 65 (1994) 151-194.

[7] Hirsch M.W.: Differential Topology, Grad. Texts Math., Vol. 33, Springer Verlag, Berlin, 1976. 
[8] Jiang H. und L. Qi: A new nonsmooth equations approach to nonlinear complementarity problems: SIAM J. Control Optim., Vol. 35, No. 1, (1997) 178-193.

[9] Jongen H.Th., P. Jonker und F. Twilt: Nonlinear Optimization in $\mathbb{R}^{n}$ II: Transversality, Flows, Parametric Aspects, Peter Lang Verlag, Frankfurt a. M., 1986.

[10] Jongen H.Th., P. Jonker und F. Twilt: On one-parameter-families of sets defined by (in)equality constraints, Nieuw Archief voor Wiskunde (3) 30 (1982) 307-322.

[11] Jongen H.Th., P. Jonker und F. Twilt: Critical sets in parametric optimization, Math. Program. 34 (1986), 333-353.

[12] Jongen, H.Th., T. Möbert, J-J. Rückmann und K. Tammer: On inertia and Schur complement in optimization. Linear Algebra Appl. 95 (1987), $97-109$.

[13] Luo Zhi-Quan, Jong-Shi Pang und Daniel Ralph: Mathematical Programs with Equilibrium Constraints, Cambridge University Press, 1996.

[14] Ouellete, D.V.: Schur complements and statistics, Lin. Alg. Appl. 36 (1981), 187-295.

[15] Robinson, S.M.: Mathematical foundations of nonsmooth embedding methods, Mathematical Programming 48 (1990) 221-229.

[16] Rückmann J.-J. and K. Tammer: On linear-quadratic perturbations in one-parametric nonlinear optimization. Systems Science 18 (1992) 1, 3748

[17] Sellami H.: A homotopy continuation method for solving normal equations, Mathematical Programming 82 (1998) 317-337.

[18] Sellami H. and S.M. Robinson: Homotopies based on nonsmooth equations for solving nonlinear variational inequalities, in G. di Pillo. F. Giannessi (Eds.), Nonlinear Optimization and Applications, Plenum Press, New York, 1996, pp. 329-343. 
[19] Sellami H. and S.M. Robinson: Implementation of a continuation method for normal maps, Mathematical Programming, Series B 76 (1997) 563-578. 